\title{
Pharmacomechanical Thrombectomy for Acute Limb Threatening Lower Extremity Arterial Thrombosis Secondary to COVID-19
}

\author{
Rajesh Girdhardas Mundhada ${ }^{1}$ Atul Dharmaraj Rewatkar ${ }^{1} \quad$ Anju Rajesh Mundhada ${ }^{1}$ \\ Aishwarya Atul Rewatkar ${ }^{1}$ Nikita Navin Chandak ${ }^{2}$
}

1 Pulse Clinic and Hospital, Nagpur, Maharashtra, India

Address for correspondence Rajesh Girdhardas Mundhada, MBBS,

2 Final Year Medical Student, King Edward Medical College, Mumbai, MD, DNB, Pulse Clinic and Hospital, Nagpur 440012, Maharashtra, Maharashtra, India India (e-mail: pulseclinic.rajesh@gmail.com).

J Clin Interv Radiol ISVIR 2022;6:170-176.

\begin{abstract}
Keywords

- COVID-19-related arterial thrombosis

- Angio-Jet thrombectomy system

Purpose The aim of this study was to report the clinical effectiveness and safety of pharmacomechanical thrombectomy (PMT) for acute limb threatening lower extremity arterial thrombosis secondary to coronavirus disease 2019 (COVID-19).

Methods In this retrospective study, the clinical and imaging records of six patients (6 men, mean age 56 years) with COVID-19-related lower extremity arterial thrombosis and treated with PMT using the Angio-jet device were reviewed. Patients presented with symptoms of acute pain in the affected limb, pallor, pulselessness, paresthesia, or paralysis. Thrombosis involved below knee arteries in all patients, and above knee arteries in three patients. Reteplase $18 \mathrm{mg}$ was infused into the thrombus using the power pulse mode on the Angio-Jet device. This was followed by rheolytic thrombectomy and then, intra-arterial infusion of urokinase $100,000 \mathrm{IU} /$ hour for 5 hours. All patients received systemic intravenous heparin. Technical success, clinical success, and complications of PMT were analyzed.

Results The mean thrombectomy duration was $190.83 \pm 99.18$ seconds. Adjuvant balloon angioplasty was performed in two patients. The mean hospital stay was $7.67 \pm 4.50$ days. PMT was technically successful in all $(100 \%)$ patients. Five patients had excellent clinical outcome with at least one palpable pulse and no tissue loss. One patient required minor amputation. One patient developed self-limiting retroperitoneal hematoma.

Conclusion In our series, PMT with supplemental intra-arterial urokinase infusion was highly successful in treating acute peripheral arterial thrombosis secondary to COVID19.
\end{abstract}

article published online January 17, 2022
DOI https://doi.org/ $10.1055 / \mathrm{s}-0041-1740459$ ISSN 2457-0214.
(C) 2022. Indian Society of Vascular and Interventional Radiology. All rights reserved.

This is an open access article published by Thieme under the terms of the Creative Commons Attribution-NonDerivative-NonCommercial-License, permitting copying and reproduction so long as the original work is given appropriate credit. Contents may not be used for commercial purposes, or adapted, remixed, transformed or built upon. (https://creativecommons.org/ licenses/by-nc-nd/4.0/)

Thieme Medical and Scientific Publishers Pvt. Ltd., A-12, 2nd Floor, Sector 2, Noida-201301 UP, India 


\section{Introduction}

The first case of SARS-Cov-2-severe acute respiratory syndrome coronavirus 2-was reported in December 2019, from Wuhan, China. Later in March 2020, the World Health Organization declared the disease as pandemic and named it as coronavirus disease 2019 (COVID-19). ${ }^{1}$ The disease presentation ranged from asymptomatic disease to lifethreatening SARS. ${ }^{2}$ A few patients were reported to have developed arterial and venous thrombosis. COVID-19 virus directly invades the endothelium through the angiotensin converting enzyme 2 receptors present on the endothelium and causes endotheliitis. This leads to localized activation of proinflammatory factors (cytokine storm) and vascular thrombosis. $^{3}$

Acute limb ischemia (ALI) is a vascular emergency marked by sudden disruption of arterial perfusion threatening the viability of the limb. Symptoms occur within 14 days of onset of the thrombotic or embolic event. ${ }^{4,5}$ Sudden stoppage of blood supply affects all metabolically active tissues such as muscles, bones, nerves, and skin, resulting in symptoms of pain, pallor, paresthesia, paralysis, poikilothermia, and skin loss. ${ }^{6}$ If the supply is not restored promptly, there is a higher risk of limb loss. The incidence of ALI ranges from 9 to 16 cases per 100,000 population per year for lower $\operatorname{limb}^{4,7,8}$ and 1 to 3 cases per 100,000 people per year for upper $\operatorname{limb}^{9}$ Despite early revascularization, 30-day mortality rate and amputation rate are between 10 and 15\%. Incidence of arterial thrombosis in COVID-19 disease is unknown. Only a few case reports and one small series have reported outcomes of interventions in patients with COVID-19-related lower extremity arterial thrombosis. ${ }^{10-13}$

In this study, we report the outcomes of pharmacomechanical thrombectomy (PMT) in six patients who presented with ALI secondary to COVID-19-related lower extremity arterial thrombosis.

\section{Methods}

\section{Study Subjects}

This is a retrospective, institutional review board approved study. Six consecutive patients (6 men, mean age $56.17 \pm 11.68$ years) with COVID-19 (detected on real-time reverse transcription-polymerase chain reaction test) and presenting with acute lower extremity ischemia to our hospital during the COVID-19 pandemic from October 1, 2020 , to February 28, 2021, were included in this study. Cases with ALI with no history of COVID-19 and nonsalvageable limb were excluded from the study.

Of the six patients, three had mild COVID-19 disease (high-resolution computed tomography [HRCT] severity scores of 4,5 , and 5 , respectively) and were treated as outpatients, while three had moderate lung involvement (HRCT severity scores were 9, 9, and 11 respectively) and required hospitalization for oxygen support. These three hospitalized patients were on prophylactic anticoagulation using enoxaparin $40 \mathrm{mg}$ subcutaneous (SC), once a day.
All patients had clinically salvageable limb at the time of presentation. The presenting symptoms were acute pain in the affected limb, pallor, pulselessness, paresthesia, or paralysis. Three patients had diabetes mellitus and systemic hypertension while three others had no comorbidities. The ankle brachial index (ABI) and toe brachial index (TBI) were not recordable at the time of presentation. The preprocedural diagnosis of ALI was achieved clinically with assistance of color Doppler or by computed tomography angiography. The location and extent of arterial occlusion on angiography is depicted in - Fig. 1. Thrombosis involved below knee arteries in all patients, and above knee arteries in three patients. All six patients had thrombosed anterior and posterior tibial arteries, peroneal artery, and occlusion of the dorsalis pedis artery and plantar arteries. The popliteal artery was involved in four patients while the superficial femoral artery was thrombosed in two patients. Common iliac, external iliac, and common femoral arteries were thrombosed in one patient each.

\section{Pharmacomechanical Thrombectomy}

Preprocedurally, patients were hydrated using intravenous (IV) normal saline at 70 to $100 \mathrm{~mL} /$ hour (depending upon cardiac status) for at least 3 hours, and the same IV hydration was continued during and after the procedure. The Angio-Jet thrombectomy system with the Solent Omni 6F catheter (Boston Scientific, Natick, Massachusetts, United States) was used in all patients. Depending on the location of the thrombus, an antegrade or a retrograde or bilateral femoral arterial access was obtained under local anesthesia and ultrasound guidance. All patients received 5,000 IU bolus of unfractionated heparin and additional 1,000 IU at every hour of the procedure. Following diagnostic angiography, a $4 \mathrm{~F}$ Head Hunter catheter and a $360 \mathrm{~cm}$ hydrophilic angled tip 0.035 wire (Terumo, Japan) were used to cross the thrombotic occlusion and the wire was kept in the plantar arch. Over the wire, the Solent Omni catheter was advanced and thrombolytics (Reteplase $18 \mathrm{mg}$ ) was sprayed in the "power pulse mode" along the entire length of the thrombus. The dwell time of the thrombolytic drug was a minimum of 60 minutes. A repeat angiogram was performed to evaluate the effect of thrombolytic drugs. Then the Omni catheter was reintroduced and the residual thrombus was removed using the "thrombectomy mode" by withdrawing or advancing the catheter at the rate of 5 to $10 \mathrm{~mm} / \mathrm{sec}$. Thrombectomy was repeated if thrombus persisted. Thrombectomy time never exceeded 480 seconds. Adjuvant procedures such as balloon angioplasty were performed in two patients. All patients received additional thrombolysis to treat microvascular thrombi, with intra-arterial infusion of urokinase at the rate of $100,000 \mathrm{IU}$ per hour along with heparin at 1,000 IU/hour for 5 hours. A check angiogram was performed to evaluate the end result.

\section{Postoperative Management}

All patients were treated with oral aspirin $75 \mathrm{mg}$ once a day, rivaroxaban $2.5 \mathrm{mg}$ twice a day, cilostazol $100 \mathrm{mg}$ twice a day, and rosuvastatin $20 \mathrm{mg} /$ day along with other supportive 


\begin{tabular}{|c|c|c|}
\hline \multicolumn{2}{|c|}{ Limb } \\
\hline \multicolumn{2}{|c|}{ CIA } \\
\hline \multicolumn{2}{|c|}{ EIA } \\
\hline \multicolumn{2}{|c}{ CFA } \\
\hline $\begin{aligned} \text { PROXIMAL } \\
\text { SFA }\end{aligned}$ \\
\hline \multicolumn{2}{|c}{ Distal SFA } \\
\hline \multicolumn{2}{|c|}{ POP A } \\
\hline A & P & P \\
T & E & T \\
A & A & A \\
\hline D & & PLA \\
P & NTA \\
A & & R \\
\hline
\end{tabular}
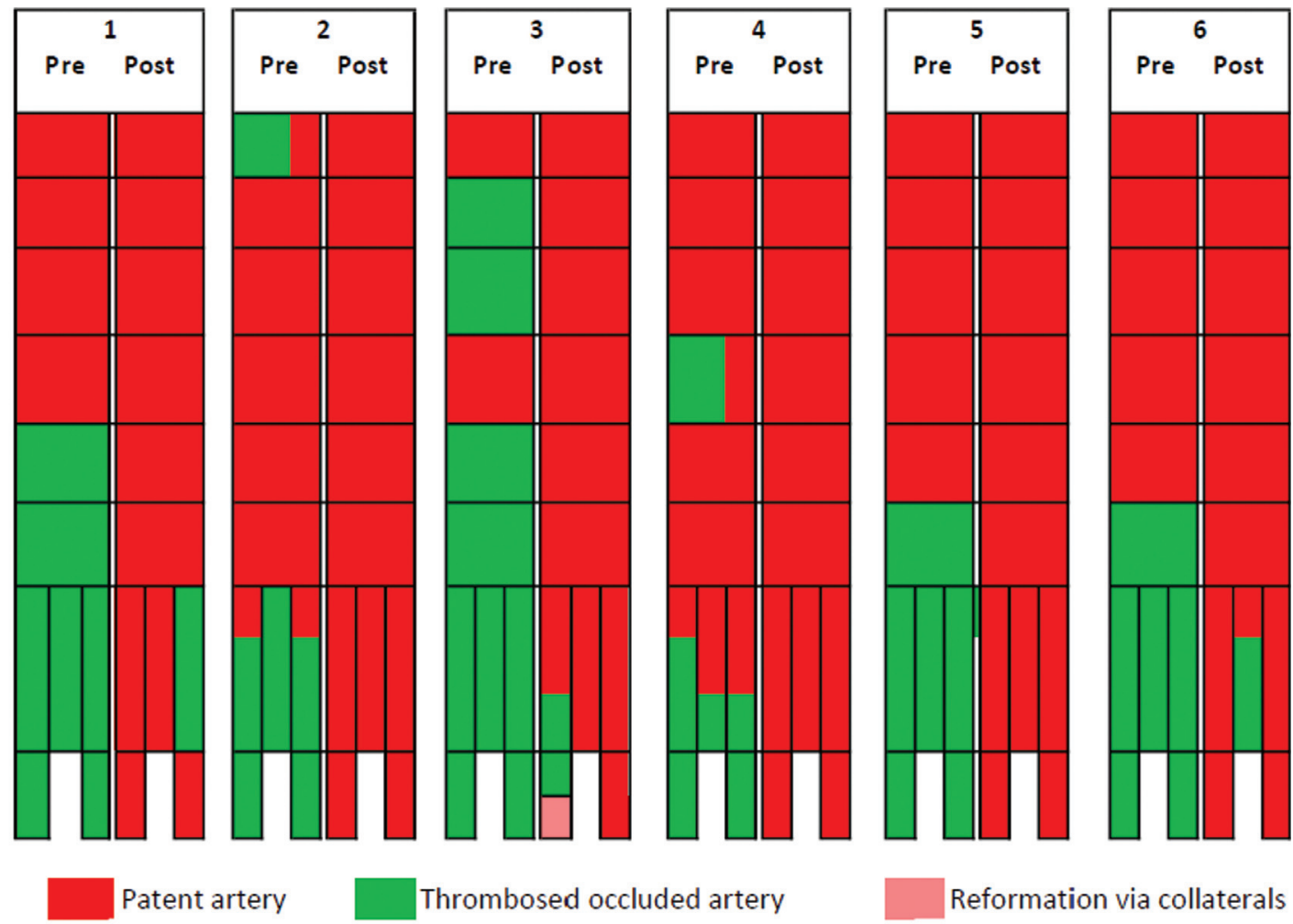

Thrombosed occluded artery

Reformation via collaterals

Pre- Pre procedural angiography

Post - Post procedural angiography

Fig. 1 Bar graph showing pre- and post-Angio-Jet thrombectomy results showing technical success in six patients of coronavirus disease 2019related lower limb arterial thrombosis causing acute limb ischemia.

treatment for COVID-19 and other comorbidities. Patients were monitored for bleeding, renal failure, and other complications.

\section{Outcomes}

The effectiveness of PMT was assessed on its technical and clinical success. The technical success was defined as angiographic visualization of previously thrombosed vessels as a direct continuity or by distal reformation in the foot. The clinical success was determined by relief of pain, restoration of pulse in the foot, improvement of motor and sensory functions, and requirement of major or minor amputation. The outcome was rated as excellent, good, fair, and poor, depending upon the clinical outcome as described in - Table 1.

\section{Results}

The mean procedural time (excluding extended thrombolysis) was $157.33 \pm 69.73$ minutes. Average dose of heparin

Table 1 Categorization of clinical outcome following pharmacomechanical thrombectomy in COVID-19-related arterial thrombosis

\begin{tabular}{|l|l|}
\hline & Clinical success criteria \\
\hline Excellent & $\begin{array}{l}\text { - No rest pains } \\
\text { - Clinical palpation of at least one pulse in the foot (dorsalis pedis artery } \pm \text { posterior tibial artery) irrespective of } \\
\end{array}$ \\
& $\begin{array}{l}\text { - Matchy mottling of the skin in the foot } \\
\text { - Patient walking without support within } 7 \text { days after treatment } \\
\text { - Patient not requiring minor amputation within } 30 \text { days after treatment }\end{array}$ \\
\hline Good & $\begin{array}{l}\text { - No rest pains } \\
\text { - Clinical palpation of at least one pulse in the foot (dorsalis pedis artery } \pm \text { posterior tibial artery) irrespective of } \\
\end{array}$ \\
& $\begin{array}{l}\text { - Matchy mottling of the skin in the foot } \\
\text { - Patient walking with support within } 7 \text { days after treatment } \\
\text { - Patient requiring minor amputation within } 30 \text { days after treatment }\end{array}$ \\
\hline
\end{tabular}


Table 1 (Continued)

\begin{tabular}{|c|c|}
\hline & Clinical success criteria \\
\hline Fair & $\begin{array}{l}\text { - No rest pain/alleviation of rest pain } \\
\text { - No clinically palpable pulsation. Collaterals preserving the foot, irrespective of patchy mottling of the skin in the } \\
\text { forefoot or in the distal phalanx of one or more finger of hand } \\
\text { - Motor movements of foot significantly lost (complete foot/ankle /wrist drop) requiring a splint to walk after } \\
7 \text { days. } \\
\text { - Ambulation requiring more than } 7 \text { days with support } \\
\text { - Patient requiring minor amputation/skin grafting/flap reconstruction/major debridement but ankle is } \\
\text { preserved with no requirement of prosthesis }\end{array}$ \\
\hline Poor & - Insufficient flow requiring major amputation or causing death of the patient within 30 days \\
\hline
\end{tabular}

Abbreviation: COVID-19, coronavirus disease 2019.
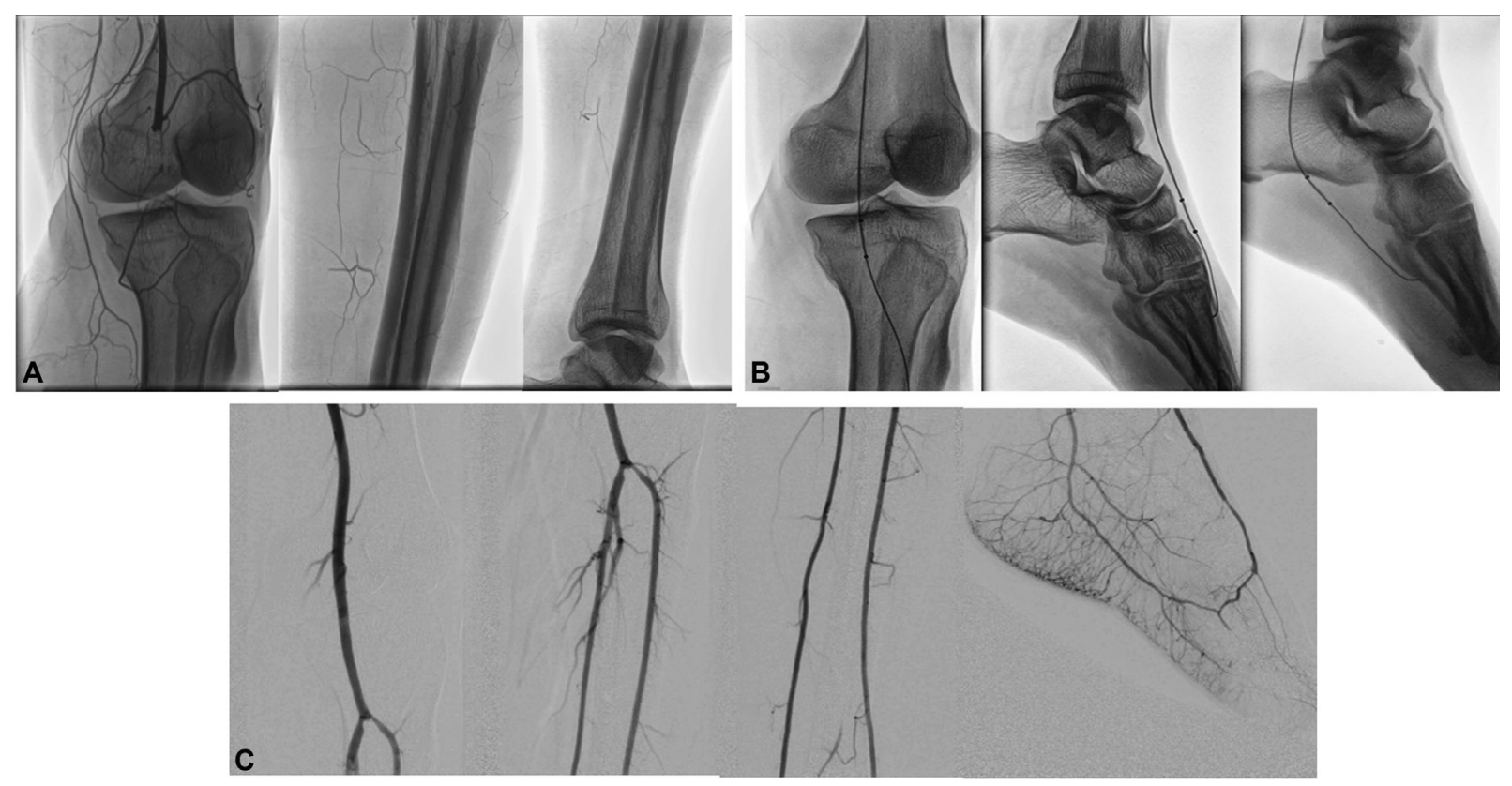

Fig. 2 (A) Preprocedure, right lower limb angiography showing thrombotic occlusion of popliteal artery and all tibial arteries with no distal reformation of foot arteries. (B) Solent Omni Angio-jet thrombectomy catheter at different arterial levels. (C) Post-Angio-Jet thrombectomy, right lower limb digital subtraction angiography showing recanalized popliteal artery, tibioperoneal trunk, posterior tibial artery, peroneal artery, anterior tibial artery, and pedal arteries along with the pedal arch.

required was $12,333.33 \pm 471.40$ units. The mean hospital stay was $7.67 \pm 4.50$ days.

The technical success of PMT was $100 \%$. On postprocedural angiography, all six (100\%) patients had adequate patency of the plantar arch (-Fig. 1, - Fig. 2, - Fig. 3). Two patients who had an underlying atherosclerotic stenosis required angioplasty. The clinical success after PMT was evaluated according to our institutional guidelines (-Table 1). Five (83.33\%) patients had excellent outcome and one (16.67\%) patient had good outcome.

One patient developed spontaneous, self-limiting, right iliopsoas hematoma on the next day, which was managed conservatively. There was no gastrointestinal, genitourinary, or intracranial bleed in any of our patients. Hemoglobinuria was seen in two (33.33\%) patients, which resolved within 24 hours after the procedure. Procedure-related anemia (mean $\mathrm{Hb}$ drop of $0.38 \pm 0.22 \mathrm{~g} / \mathrm{dL}$ ) was seen in all patients, with no patients requiring blood transfusions after the procedure. None of the patients developed renal failure.
Reperfusion edema was minimal and none of the patient required fasciotomy.

\section{Patient Follow-Up}

The average follow-up was $167.67 \pm 53.33$ days. During the follow-up, there were no deaths as of May 31, 2021. Color Doppler did not show any reocclusion of the recanalized arteries. Postprocedural ABI/TBI was normal in all six patients at 3 months of follow-up. No patient had rethrombosis or reocclusion requiring repeat intervention during the follow-up period. Only one patient required amputation of the little toe. No further tissue necrosis, amputation, or death was noted.

\section{Discussion}

COVID-19 differs from other viral infectious diseases in many ways. Arterial thrombosis is an extremely rare complication 

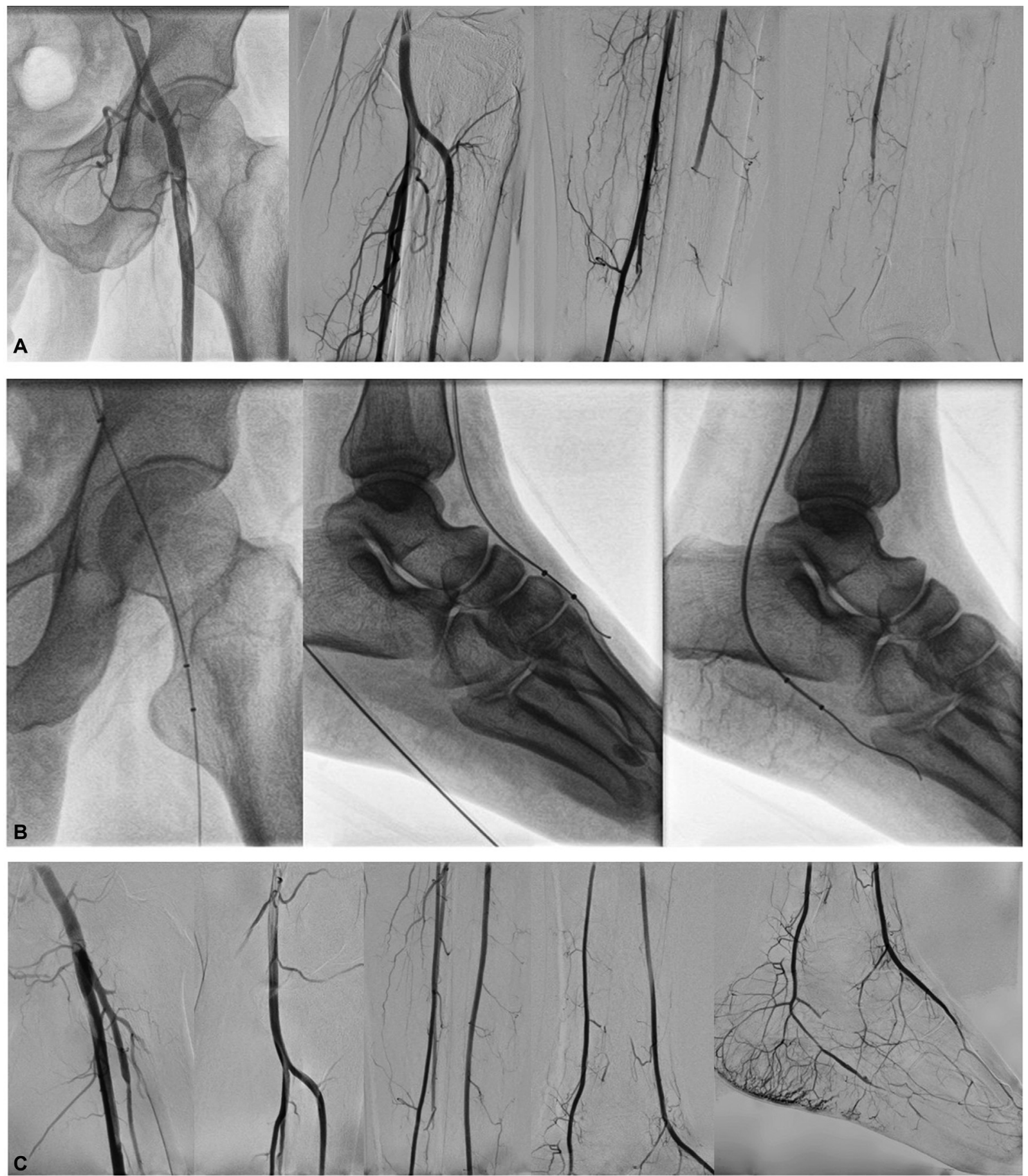

Fig. 3 (A) Preprocedure, left lower angiography showing partial thrombus in proximal superficial femoral artery with thrombotic occlusion of profunda femoris artery and tibial arteries with no distal reformation of foot arteries. (B) Solent Omni Angio-Jet thrombectomy catheter in thrombosed superficial femoral artery, dorsalis pedis artery, and plantar artery. (C) Post-Angio-jet thrombectomy, left lower limb digital subtraction angiography showing recanalized arteries with patent pedal arteries.

of other infectious diseases. Intact endothelium maintains the delicate balance between procoagulants and anticoagulants in the blood. COVID-19 virus invades the endothelium leading to inflammatory damage of the endothelium causing platelet activation, aggregation, and "in situ" thrombus formation. Arterial thrombosis in COVID-19 frequently involves young men, large arteries (aorta, mesenteric vessels, and large peripheral vessels) without preexisting arterial atherosclerotic disease. There is a lack of abnormality in coagulation parameters in COVID-19 infection. The classical parameters of disseminated intravascular coagulation, such as low platelet count, prolonged prothrombin time, and fibrinogen consumptions, are either mild or lacking in COVID-19. ${ }^{3}$

Incidence of COVID-19-related arterial thrombosis was reported to be $4.4 \%$ in critically ill patients. ${ }^{14}$ In our study, 
extensive arterial thrombosis was noted in three patients with mild COVID-19 infection who were being home quarantined and in three patients having moderate disease requiring hospitalization for oxygen support. None of our patients required intensive care unit admission for their COVID-19 disease, suggesting arterial thrombosis can happen even in mild and moderate diseases. Three patients who were treated as outpatients received no anticoagulation therapy, while the remaining three hospitalized patients received prophylactic anticoagulation. Few studies reported arterial thrombosis in COVID-19 patients despite adequate prophylactic anticoagulation (enoxaparin $40 \mathrm{mg} \mathrm{SC}$, once a day) and advocated for therapeutic anticoagulation to prevent arterial thrombosis. Some reports have emphasized the role of intermediate intensity anticoagulation (enoxaparin $0.5 \mathrm{mg} / \mathrm{kg}$, SC, once a day) for prevention of arterial thrombosis. $^{3}$

In non-COVID-19 patients, arterial thrombosis commonly affects men more than women. ${ }^{15,16}$ This may be due to the differences in the genetic and hormonal profiles of two sexes and favorable immune modulation by female sex hormones. ${ }^{15-17}$ In our study, all affected patients were men. It is not clear whether female sex offers immunity to arterial thrombosis in COVID-19.

Despite the extensive distal arterial thrombosis, treatment by PMT was very successful in these patients. Despite skin mottling and early motor loss, PMT provided excellent outcomes. Adjunctive procedures such as balloon angioplasty with or without stenting and extended intra-arterial thrombolysis to treat the residual microvascular thrombi should be considered. There was no incidence of immediate rethrombosis, which may be due to a decrease in the viral load during the treatment or due to the combined use of aspirin and rivaroxaban.

Hemoglobinuria was seen in two (33.33\%) patients. Low incidence of hemoglobinuria in our series was probably because of thrombotic occlusion of distal and small caliber arteries, having less clot burden requiring less thrombectomy duration and, so, less hemolysis. Hemoglobinuria did not result in impairment of renal function, probably secondary to adequate pre-, during-, and postprocedure IV hydration.

Delay in treatment of ALI results in high mortality and morbidity. Despite starting treatment within 12 hours of onset of ischemia, mortality and limb salvage rates are 19 and $93 \%$, respectively. ${ }^{18}$ With further delay in treatment of more than 12 hours of onset of ischemia, mortality and limb salvage rates further worsen to 31 and $78 \%$, respectively. ${ }^{19,20}$ In our series, the mortality and limb salvage rates were 0 and $100 \%$, respectively, even after delayed presentation of 24 to 96 hours. Arterial thrombosis in COVID-19 infection can involve one or more arteries simultaneously and is due to endotheliitis, causing patchy thrombosis that propagates slowly to become extensive and gives more time for treatment after the onset of ischemia.

There are many limitations to our study. Our study is retrospective and has a very small sample size. Despite the small size, our study proved the effectiveness of PMT in COVID19-related peripheral arterial ischemia. The follow-up was short, but adequate to demonstrate lack of rethrombosis in the short term. A larger prospective study with longer followup would be desirable to affirm our findings.

\section{Conclusion}

PMT is safe and effective in acute limb threatening arterial thrombosis secondary to COVID-19 infection. Rheolytic thrombectomy along with extended intra-arterial pharmacological thrombolysis could restore perfusion quickly leading to limb and life salvage.

\section{Ethical statement}

This is a retrospective, institutional review board approved study.

\section{References}

1 Mahmoud BM, Isaac NN, et al. Thromboembolism risk of COVID19 is high and associated with a higher risk of mortality: a systematic review and meta-analysis. E Clin Med (Northfield Ill) 2020;29-30:100639 PMCID: PMC7679115. Doi: 10.1016/j. eclinm.2020.100639

2 Huang C, Wang Y, Li X, et al. Clinical features of patients infected with 2019 novel coronavirus in Wuhan, China. Lancet 2020;395 (10223):497-506

3 Moroni F, Baldetti L. COVID-19 and arterial thrombosis: a potentially fatal combination. Int J Cardiol 2021;322:286-290

4 Creager MA, Kaufman JA, Conte MS. Clinical practice. Acute limb ischemia. N Engl J Med 2012;366(23):2198-2206

5 Eliason JL, Wainess RM, Proctor MC, et al. A national and single institutional experience in the contemporary treatment of acute lower extremity ischemia. Ann Surg 2003;238(03):382-389, discussion 389-390

6 Dan-Mircea O, Agata S, et al. Acute limb ischemia: an update on diagnosis and management. J Clin Med (Lond) 2019;8:1215. Doi: $10.3390 / \mathrm{jcm} 8081215$

7 Dryjski M, Swedenborg J. Acute ischemia of the extremities in a metropolitan area during one year. J Cardiovasc Surg (Torino) 1984;25(06):518-522

8 Davies B, Braithwaite BD, Birch PA, Poskitt KR, Heather BP, Earnshaw JJ. Acute leg ischaemia in Gloucestershire. Br J Surg 1997;84 (04):504-508

9 Eyers P, Earnshaw JJ. Acute non-traumatic arm ischaemia. Br J Surg 1998;85(10):1340-1346

10 Topcu AC, Ariturk C, Yilmaz E. Acute limb ischemia in a COVID-19 patient. Thromb Update 2021;2:100031. Doi: 10.1016/j. tru.2020.100031

11 Singh B, Kaur P, Ajdir N, Gupta S, Maroules M. Covid-19 presenting as acute limb ischemia. Cureus 2020;12(07):e9344. Doi: 10.7759/ cureus.9344

12 Kaur P, Posimreddy S, Singh B, et al. COVID-19 presenting as acute limb ischaemia. Eur J Case Rep Intern Med 2020;7(06):001724. Doi: 10.12890/2020_001724

13 Bellosta R, Luzzani L, Natalini G, et al. Acute limb ischemia in patients with COVID-19 pneumonia. J Vasc Surg 2020;72(06): 1864-1872

14 Cheruiyot I, Kipkorir V, Ngure B, Misiani M, Munguti J, Ogeng'o J. Arterial thrombosis in coronavirus disease 2019 patients: a rapid systematic review. Ann Vasc Surg 2021;70:273-281. Doi: 10.1016/j.avsg.2020.08.087 
176 Pharmacomechanical Thrombectomy for Acute Limb Mundhada et al.

15 Lansky AJ, Ng VG, Maehara A, et al. Gender and the extent of coronary atherosclerosis, plaque composition, and clinical outcomes in acute coronary syndromes. JACC Cardiovasc Imaging 2012;5(3, Suppl):S62-S72

16 Man JJ, Beckman JA, Jaffe IZ. Sex as a biological variable in atherosclerosis. Circ Res 2020;126(09):1297-1319

17 Fairweather D. Sex differences in inflammation during atherosclerosis. Clin Med Insights Cardiol 2015;8(Suppl 3):49-59
18 Jeffrey A, Gregory M. Choosing the correct therapeutic option for acute limb ischemia. Interv Cardiol (Lond) 2011;3(03):381-389

19 Abbott WM, Maloney RD, McCabe CC, Lee CE, Wirthlin LS. Arterial embolism: a 44 year perspective. Am J Surg 1982;143(04): 460-464

20 Elliott JPJr, Hageman JH, Szilagyi E, Ramakrishnan V, Bravo JJ, Smith RF. Arterial embolization: problems of source, multiplicity, recurrence, and delayed treatment. Surgery 1980;88(06):833-845 